\title{
THREE DIMENSIONAL SYSTEM OF GLOBALLY MODIFIED NAVIER-STOKES EQUATIONS WITH DELAY
}

\author{
TOMÁS CARABALLO, JOSÉ REAL \\ Dpto. Ecuaciones Diferenciales y Análisis Numérico \\ Universidad de Sevilla \\ Apdo. de Correos 1160 \\ 41080-Sevilla (Spain) \\ E-mails: caraball@us.es,jreal@us.es \\ ANTONIO M. MÁRQUEZ \\ Dpto. de Economía, Métodos Cuantitativos e Historia Económica \\ Universidad Pablo de Olavide \\ Sevilla (Spain) \\ E-mail: ammardur@upo.es
}

To the Memory of Professor Valery S. Melnik

\begin{abstract}
We prove existence and uniqueness of strong solutions of a three dimensional system of globally modified Navier-Stokes equations with delay in the locally Lipschitz case. The asymptotic behaviour of solutions, and the existence of pullback attractor are also analyzed.

Keywords: 3-dimensional Navier-Stokes equations, Galerkin approximations, weak solutions, existence and uniqueness of strong solutions, global attractors.
\end{abstract}

Mathematics Subject Classifications (2000): 35Q30, 35K90, 37L30

\section{Introduction}

$$
\left\{\begin{array}{l}
\frac{\partial u}{\partial t}-\nu \Delta u+(u \cdot \nabla) u+\nabla p=f(t) \\
\quad \text { in }(0,+\infty) \times \Omega, \\
\nabla \cdot u=0 \text { in }(0,+\infty) \times \Omega, \\
u=0 \text { on }(0,+\infty) \times \Gamma, \\
u(0, x)=u^{0}(x), \quad x \in \Omega,
\end{array}\right.
$$

Let $\Omega \subset \mathbb{R}^{3}$ be an open bounded set with regular boundary $\Gamma$, and consider the Navier-Stokes equations (NSE) on $\Omega$ with a homogeneous Dirichlet boundary condition where $\nu>0$ is the kinematic viscosity, $u$ is the velocity field of the fluid, $p$ the pressure, $u^{0}$ the initial velocity field, and $f(t)$ a given external force field. 
There have been many modifications of the Navier-Stokes equations, starting with Leray and mostly involving the nonlinear term, see the review paper of Constantin [Constantin, 2003]. A system, called the globally modified Navier-Stokes equations (GMNSE), which was introduced recently by Caraballo et al. [Caraballo et al., 2006] will be considered here.

We define $F_{N}: \mathbb{R}^{+} \rightarrow \mathbb{R}^{+}$by

$$
F_{N}(r):=\min \left\{1, \frac{N}{r}\right\}, \quad r \in \mathbb{R}^{+},
$$

for some $N \in \mathbb{R}^{+}$and will consider the following system of globally modified Navier-Stokes equations (GMNSE)

$$
\left\{\begin{array}{l}
\frac{\partial u}{\partial t}-\nu \Delta u+F_{N}(\|u\|)[(u \cdot \nabla) u]+\nabla p=f(t) \\
\quad \text { in }(0,+\infty) \times \Omega, \\
\nabla \cdot u=0 \text { in }(0,+\infty) \times \Omega, \\
u=0 \text { on }(0,+\infty) \times \Gamma, \\
u(0, x)=u^{0}(x), \quad x \in \Omega,
\end{array}\right.
$$

The GMNSE (2) are indeed globally modified - the modifying factor $F_{N}(\|u\|)$ depends on the norm $\|u\|=\|\nabla u\|_{\left(L^{2}(\Omega)\right)^{3 \times 3} \text {, which in turn depends }}$ on $\nabla u$ over the whole domain $\Omega$ and not just at or near the point $x \in \Omega$ under consideration. Essentially, it prevents large gradients dominating the dynamics and leading to explosions. It violates the basic laws of mechanics, but mathematically the GMNSE (2) are a well defined system of equations, just like the modified versions of the NSE of Leray and others with other mollifications of the nonlinear term, see the review paper [Constantin, 2003]. It is worth mentioning that a global cut off function involving the $D\left(A^{1 / 4}\right)$ norm for the two dimensional stochastic Navier-Stokes equations is used in [Flandoli \& Maslowski, 1995], and a cut-off function similar to the one we will use here was considered in [Yoshida \& Giga, 1984].

As we have already mentioned, the GMNSE (2) has been introduced and studied in [Caraballo et al., 2006] (see also [Caraballo et al., 2008], [Kloeden et al., 2007],
[Romito, 2009], [Kloeden et al., 2009b], [Kloeden \& Valero, 2007] and the review paper [Kloeden et al., 2009a]).

However, there are many situations in which one can consider that the model is better described if we allow some delay in the equations. These situations may appear, for instance, when we want to control the system by applying a force which takes into account not only the present state of the system but the history of the solutions. Therefore, in this paper we are interested in the case in which terms containing finite delays appear. Namely, we consider the following version of GMNSE (we will refer to it as GMNSED):

$$
\left\{\begin{array}{l}
\frac{\partial u}{\partial t}-\nu \Delta u+F_{N}(\|u\|)[(u \cdot \nabla) u]+\nabla p \\
=G(t, u(t-\rho(t))) \quad \text { in }(\tau,+\infty) \times \Omega, \\
\nabla \cdot u=0 \text { in }(\tau,+\infty) \times \Omega, \\
u=0 \text { on }(\tau,+\infty) \times \Gamma, \\
u(\tau, x)=u^{0}(x), \quad x \in \Omega, \\
u(t, x)=\phi(t-\tau, x), \text { in }(\tau-h, \tau) \times \Omega,
\end{array}\right.
$$

where $\tau \in \mathbb{R}$ is an initial time, the term $G(t, u(t-\rho(t)))$ is an external force depending eventually on the value $u(t-\rho(t)), \rho(t) \geq 0$ is a delay function and $\phi$ is a given velocity field defined in $(-h, 0)$, with $h>0$ a fixed time such that $\rho(t) \leq h$.

In the next section we state some preliminaries and establish the framework for our problem. Section 3 is devoted to the existence and uniqueness of weak and strong solutions of our problem. In Section 4 we analyze the asymptotic behaviour of solutions, which is completed in the final section by proving the existence of pullback attractor for our model. It is worth mentioning that as the delay model is non-autonomous, the classical theory of global attractors is not appropriate to handle this problem, unless the non-autonomous term possesses a special form. However, the theory of pullback attractors allows for more general non-autonomous terms. 


\section{Preliminaries}

To set our problem in the abstract framework, we consider the following usual abstract spaces (see [Lions, 1969] and [Temam, 1977, Temam, 1995]):

$$
\mathcal{V}=\left\{u \in\left(C_{0}^{\infty}(\Omega)\right)^{3}: \operatorname{div} u=0\right\},
$$

$H=$ the closure of $\mathcal{V}$ in $\left(L^{2}(\Omega)\right)^{3}$ with inner product $(\cdot, \cdot)$ and associate norm $|\cdot|$, where for $u, v \in$ $\left(L^{2}(\Omega)\right)^{3}$,

$$
(u, v)=\sum_{j=1}^{3} \int_{\Omega} u_{j}(x) v_{j}(x) \mathrm{d} x,
$$

$V=$ the closure of $\mathcal{V}$ in $\left(H_{0}^{1}(\Omega)\right)^{3}$ with scalar product $((\cdot, \cdot))$ and associate norm $\|\cdot\|$, where for $u, v \in\left(H_{0}^{1}(\Omega)\right)^{3}$,

$$
((u, v))=\sum_{i, j=1}^{3} \int_{\Omega} \frac{\partial u_{j}}{\partial x_{i}} \frac{\partial v_{j}}{\partial x_{i}} \mathrm{~d} x .
$$

It follows that $V \subset H \equiv H^{\prime} \subset V^{\prime}$, where the injections are dense and compact. Finally, we will use $\|\cdot\|_{*}$ for the norm in $V^{\prime}$ and $\langle\cdot, \cdot\rangle$ for the duality pairing between $V$ and $V^{\prime}$.

Now we define the trilinear form $b$ on $V \times V \times V$ by

$$
b(u, v, w)=\sum_{i, j=1}^{3} \int_{\Omega} u_{i} \frac{\partial v_{j}}{\partial x_{i}} w_{j} \mathrm{~d} x, \quad \forall u, v, w \in V,
$$

and we denote

$$
b_{N}(u, v, w)=F_{N}(\|v\|) b(u, v, w), \quad \forall u, v, w \in V .
$$

The form $b_{N}$ is linear in $u$ and $w$, but it is nonlinear in $v$. Evidently we have $b_{N}(u, v, v)=0$, for all $u, v \in V$. Moreover, from the properties of $b$ (see [Robinson, 2001] or [Temam, 1977]), and the definition of $F_{N}$, one easily obtains the existence of a constant $C_{1}>0$ only dependent on $\Omega$ such that

$$
\left|b_{N}(u, v, w)\right| \leq N C_{1}\|u\|\|w\|, \quad \forall u, v, w \in V .
$$

Thus, if we denote

$$
\left\langle B_{N}(u, v), w\right\rangle=b_{N}(u, v, w), \quad \forall u, v, w \in V,
$$

we have

$$
\left\|B_{N}(u, v)\right\|_{*} \leq N C_{1}\|u\|, \quad \forall u, v \in V .
$$

We also consider $A: V \rightarrow V^{\prime}$ defined by $\langle A u, v\rangle=((u, v))$. Denoting $D(A)=\left(H^{2}(\Omega)\right)^{3} \cap V$, then $A u=-P \Delta u, \forall u \in D(A)$, is the Stokes operator $\left(P\right.$ is the ortho-projector from $\left(L^{2}(\Omega)\right)^{3}$ onto $H)$.

We recall (see [Temam, 1977]) that there exists a constant $C_{2}>0$ depending only on $\Omega$ such that

$$
|b(u, v, w)| \leq C_{2}\|u\|^{1 / 2}|A u|^{1 / 2}\|v\||| w \mid,
$$

for all $u \in D(A), v \in V, w \in H$, and

$$
|b(u, v, w)| \leq\left. C_{2}\|u\|\|v\||| w\right|^{1 / 2}\|w\|^{1 / 2},
$$

for all $u, v, w \in V$. (See [Romito, 2009] for the proof of $(6)$ ).

Moreover, we assume $G: \mathbb{R} \times H \longrightarrow H$ is such that

c1) $G(\cdot, u): \mathbb{R} \longrightarrow H$ is measurable, $\forall u \in H$,

c2) there exists nonnegative function $g \in L_{l o c}^{p}(\mathbb{R})$ for some $1 \leq p \leq+\infty$, and a nondecreasing function $L:(0, \infty) \rightarrow(0, \infty)$, such that for all $R>0$ if $|u|,|v| \leq R$, then

$$
|G(t, u)-G(t, v)| \leq L(R) g^{1 / 2}(t)|u-v|,
$$

for all $t \in \mathbb{R}$, and

c3) there exists a nonnegative function $f \in$ $L_{l o c}^{1}(\mathbb{R})$, such that for any $u \in H$,

$$
|G(t, u)|^{2} \leq g(t)|u|^{2}+f(t), \quad \forall t \in \mathbb{R} .
$$

Finally, we suppose $\phi \in L^{2 p^{\prime}}(-h, 0 ; H)$ and $u^{0} \in H$, where $\frac{1}{p}+\frac{1}{p^{\prime}}=1$.

In this situation, we consider a delay function $\rho \in$ $C^{1}(\mathbb{R})$ such that $0 \leq \rho(t) \leq h$ for all $t \in \mathbb{R}$, and there exists a constant $\rho_{*}$ satisfying

$$
\rho^{\prime}(t) \leq \rho_{*}<1 \quad \forall t \in \mathbb{R} .
$$

Definition 2.1. Let $\tau \in \mathbb{R}, u^{0} \in H$ and $\phi \in$ $L^{2 p^{\prime}}(-h, 0 ; H)$ be given. A weak solution of (3) is a function

$$
u \in L^{2 p^{\prime}}(\tau-h, T ; H) \cap L^{2}(\tau, T ; V) \cap L^{\infty}(\tau, T ; H)
$$

for all $T>\tau$, such that

$$
\left\{\begin{aligned}
\frac{\mathrm{d}}{\mathrm{d} t} u(t) & +\nu A u(t)+B_{N}(u(t), u(t)) \\
& =G(t, u(t-\rho(t))) \text { in } \mathcal{D}^{\prime}\left(\tau,+\infty ; V^{\prime}\right), \\
u(\tau) & =u^{0} \\
u(t) & =\phi(t-\tau) \quad t \in(\tau-h, \tau)
\end{aligned}\right.
$$


or equivalently

$$
\begin{aligned}
& (u(t), w)+\nu \int_{\tau}^{t}((u(s), w)) d s \\
& +\int_{\tau}^{t} b_{N}(u(s), u(s), w) d s=\left(u^{0}, w\right) \\
& \quad+\int_{\tau}^{t}(G(s, u(s-\rho(s))), w) d s
\end{aligned}
$$

for all $t \geq \tau$ and all $w \in V$, and coincides with $\phi(t)$ in $(\tau-h, \tau)$.

Remark 2.2. If $u$ is a weak solution of (3) and we define $\tilde{g}(t)=g \circ \theta^{-1}(t)$, where $\theta:[\tau,+\infty) \longrightarrow$ $[\tau-\rho(\tau),+\infty)$ is the differentiable and strictly increasing function given by $\theta(s)=s-\rho(s)$, we obtain

$$
\begin{aligned}
& \int_{\tau}^{T}|G(t, u(t-\rho(t)))|^{2} d t \\
& \leq \int_{\tau}^{T} g(t)|u(t-\rho(t))|^{2} d t+\int_{\tau}^{T} f(t) d t \\
& \leq \frac{1}{1-\rho_{*}} \int_{\tau-\rho(\tau)}^{T-\rho(T)} \tilde{g}(t)|u(t)|^{2} d t+\int_{\tau}^{T} f(t) d t \\
& \leq \frac{1}{1-\rho_{*}} \int_{\tau-\rho(\tau)}^{T} \tilde{g}(t)|u(t)|^{2} d t+\int_{\tau}^{T} f(t) d t,
\end{aligned}
$$

and therefore, taking into account that $\tilde{g} \in L^{p}(\tau-$ $\rho(\tau), T)$ for all $T>\tau$, we have that $G(t, u(t-\rho(t)))$ belongs to $L^{2}(\tau, T ; H)$ for all $T>\tau$.

Thus, if $u \in L^{2}(\tau, T ; V)$ for all $T>\tau$ and satisfies the equation

$\frac{\mathrm{d}}{\mathrm{d} t} u(t)+\nu A u(t)+B_{N}(u(t), u(t))=G(t, u(t-\rho(t)))$,

in $\mathcal{D}^{\prime}\left(\tau,+\infty ; V^{\prime}\right)$, then, as a consequence of (4), $\frac{\mathrm{d}}{\mathrm{d} t} u(t) \in L^{2}\left(\tau, T ; V^{\prime}\right)$, and consequently (see [Temam, 1995]) $u \in C([\tau,+\infty) ; H)$ and satisfies the energy equality, for all $\tau \leq s \leq t$,

$$
\begin{gathered}
|u(t)|^{2}-|u(s)|^{2}+2 \nu \int_{s}^{t}\|u(r)\|^{2} d r \\
\quad=2 \int_{s}^{t}(G(r, u(r-\rho(r))), u(r)) d r .
\end{gathered}
$$

We will prove the existence of (strong) solutions in the next section. First, we will prove the uniqueness of weak solutions for our model in a similar way as Romito [Romito, 2009] did for the model without delay. We will only include the detailed estimates which involve the delay term.

Theorem 2.3. Under the preceding assumptions, there exists at most a weak solution $u$ of (3).

The proof is similar to, but a bit more complicated than in the 2D-NSE case and depends on the following Lemma.

Lemma 2.4. ([Romito, 2009]) For every $u, v \in V$, and each $N>0$,

1. $0 \leq\|u\| F_{N}(\|u\|) \leq N$,

2. $\left|F_{N}(\|u\|)-F_{N}(\|v\|)\right|$ $\leq \frac{1}{N} F_{N}(\|u\|) F_{N}(\|v\|)\|u-v\|$.

Proof of Theorem 2.3: $\quad$ Let $u, v$ be two weak solutions with the same initial conditions and set $w=$ $v-u$. Then, using the energy equality, we obtain

$$
\begin{aligned}
& \frac{1}{2} \frac{\mathrm{d}}{\mathrm{d} t}|w|^{2}+\nu\|w\|^{2}+\langle\mathcal{N} \mathcal{L}(u, v), w\rangle \\
& =(G(t, v(t-\rho(t)))-G(t, u(t-\rho(t))), w),
\end{aligned}
$$

where we have set $\langle\mathcal{N} \mathcal{L}(u, v), w\rangle=$ $F_{N}(\|u\|) b(u, u, w)-F_{N}(\|v\|) b(v, v, w)$. From the properties of the trilinear form $b$ it easily follows that

$$
\begin{aligned}
& \langle\mathcal{N} \mathcal{L}(u, v), w\rangle=F_{N}(\|u\|) b(w, u, w) \\
& +\left(F_{N}(\|u\|)-F_{N}(\|v\|)\right) b(v, u, w) \\
& \quad+F_{N}(\|v\|) b(v, w, w) .
\end{aligned}
$$

Now using Lemma 2.4, formula (6) and Young's inequality (see [Romito, 2009] for the details) there exists a constant $C_{3}>0$, which depends on $C_{2}$ and $\nu$, such that ,

$$
\langle\mathcal{N} \mathcal{L}(u, v), w\rangle \leq \nu\|w\|^{2}+C_{3} N^{4}|w|^{2}
$$

Consequently, we obtain

$$
\begin{aligned}
& \frac{\mathrm{d}}{\mathrm{d} t}|w|^{2} \leq 2 C_{3} N^{4}|w|^{2} \\
& +2(G(t, v(t-\rho(t)))-G(t, u(t-\rho(t))), w) .
\end{aligned}
$$

Let us now estimate the last term in (13). For a fixed $T>\tau$, we know that $u$ and $v$ belong to 
$C([\tau, T] ; H)$, thus there exists $R_{T}>0$ such that $|u(s)| \leq R_{T}$ and $|v(s)| \leq R_{T}$, for all $s \in[\tau, T]$. Consequently, by $\mathrm{c} 2$ ) and the fact that $w=0$ in $(\tau-h, \tau)$, it is not difficult to obtain

$$
\begin{aligned}
& 2 \int_{\tau}^{t}(G(s, v(s-\rho(s)))-G(s, u(s-\rho(s))), w(s)) d s \\
& \leq \frac{2 L\left(R_{T}\right)}{\left(1-\rho_{*}\right)^{1 / 2}} \int_{\tau}^{t}(\tilde{g}(s)+1)|w(s)|^{2} d s
\end{aligned}
$$

for all $t \in[\tau, T]$.

Thus we obtain

$$
\frac{d}{d t}|w|^{2} \leq\left[2 C_{3} N^{4}+\frac{2 L\left(R_{T}\right)}{\left(1-\rho_{*}\right)^{1 / 2}}(\tilde{g}(t)+1)\right]|w|^{2},
$$

in $[\tau, T]$, and the result follows from the Gronwall lemma, since $|w(0)|^{2}=0$.

\section{Existence and uniqueness of weak and strong solutions}

In the previous section, we proved the uniqueness of weak solutions for our model. In the following theorem, we will prove the existence (and therefore uniqueness) of weak and/or strong solutions.

Theorem 3.1. Under the conditions c1)-c3) in the previous section, assume that $\tau \in \mathbb{R}, u^{0} \in H$ and $\phi \in L^{2 p^{\prime}}(-h, 0 ; H)$ are given. Then, there exists a unique weak solution $u$ of (3) which is, in fact, a strong solution in the sense that

$$
u \in C([\tau+\varepsilon, T] ; V) \cap L^{2}(\tau+\varepsilon, T ; D(A)),
$$

for all $T-\tau>\varepsilon>0$.

Moreover, if $u^{0} \in V$, then

$$
u \in C([\tau, T] ; V) \cap L^{2}(\tau, T ; D(A)),
$$

for all $T>\tau$.

Proof. For simplicity, and without loss of generality, we assume $\tau=0$.

Consider the Galerkin approximations for the GMNSED, given by

$$
\left\{\begin{array}{c}
\frac{\mathrm{d} u_{m}}{\mathrm{~d} t}+\nu A u_{m}+P_{m} B_{N}\left(u_{m}, u_{m}\right) \\
=P_{m} G\left(t, u_{m}(t-\rho(t))\right) \\
u_{m}(0)=P_{m} u^{0}, u_{m}=P_{m} \phi \text { in }(-h, 0),
\end{array}\right.
$$

where $u_{m}=\sum_{j=1}^{m} u_{m, j} e_{j}, A u_{m}=\sum_{j=1}^{m} \lambda_{j} u_{m, j} e_{j}$. Here the $\lambda_{j}$ and $e_{j}$ are the corresponding eigenvalues and orthonormal eigenfunctions of the operator $A$ and $P_{m}$ is the projection onto the subspace of $H$ spanned by $\left\{e_{1}, \ldots, e_{m}\right\}$. Then

$$
\left\|u_{m}\right\|^{2}=\sum_{j=1}^{m} \lambda_{j} u_{m, j}^{2}, \quad\left|A u_{m}\right|^{2}=\sum_{j=1}^{m} \lambda_{j}^{2} u_{m, j}^{2} .
$$

In addition

$$
\left|u_{m}\right|^{2}=\sum_{j=1}^{m} u_{m, j}^{2},
$$

which can be interpreted as either the Euclidean norm of $u_{m} \in \mathbb{R}^{m}$ or the $L^{2}$-norm of $u_{m} \in H$.

From the assumptions on $A, B_{N}$ and $G$, we know that there exists a local solution $u_{m}$ of (17) defined in $\left[0, t^{m}\right)$, with $0<t^{m} \leq+\infty$ (see for example [Hale \& Lunel, 1993]). The uniqueness of solution of (17) can be proved as in Theorem 2.3, and the fact that the local solution is a global one is a consequence of the estimate (18) below.

Let us fix $0<T<t_{m}$. It is standard that if we take the inner product of the Galerkin ODE (17) with $u_{m}$ and use that $b\left(u_{m}, u_{m}, u_{m}\right)=0$, we obtain

$$
\frac{1}{2} \frac{\mathrm{d}}{\mathrm{d} t}\left|u_{m}\right|^{2}+\nu\left\|u_{m}\right\|^{2}=\left(G\left(t, u_{m}(t-\rho(t))\right), u_{m}\right),
$$

and taking into account $\mathrm{c} 3$ ) and that $\lambda_{1}\left|u_{m}\right|^{2} \leq$ $\left\|u_{m}\right\|^{2}$,

$$
\begin{aligned}
& \frac{\mathrm{d}}{\mathrm{d} t}\left|u_{m}\right|^{2}+\nu\left\|u_{m}\right\|^{2} \\
& \quad \leq \frac{1}{\nu \lambda_{1}}\left(g(t)\left|u_{m}(t-\rho(t))\right|^{2}+f(t)\right) .
\end{aligned}
$$

Consequently, integrating between 0 and $t, t \leq T$, and using the function $\tilde{g}$ defined in Remark 2.2, we obtain

$$
\begin{aligned}
&\left|u_{m}(t)\right|^{2}+\nu \int_{0}^{t}\left\|u_{m}(s)\right\|^{2} d s \leq\left|u^{0}\right|^{2} \\
&+ \frac{1}{\nu \lambda_{1}} \int_{0}^{t}\left(g(s)\left|u_{m}(s-\rho(s))\right|^{2}+f(s)\right) d s \\
& \quad \leq K_{T}+\frac{1}{\nu \lambda_{1}\left(1-\rho_{*}\right)} \int_{0}^{t} \tilde{g}(s)\left|u_{m}(s)\right|^{2} d s
\end{aligned}
$$

for all $t \in[0, T]$, where $K_{T}=\left|u^{0}\right|^{2}+$ $\frac{1}{\nu \lambda_{1}\left(1-\rho_{*}\right)} \int_{-\rho(0)}^{0} \tilde{g}(s)|\phi(s)|^{2} d s+\frac{1}{\nu \lambda_{1}} \int_{0}^{T} f(s) d s$. 
Thus, by the Gronwall lemma,

$$
\begin{aligned}
\left|u_{m}(t)\right|^{2} & \leq K_{T} \exp \left(\frac{1}{\nu \lambda_{1}\left(1-\rho_{*}\right)} \int_{0}^{T} \tilde{g}(s) d s\right) \\
& =C_{T},
\end{aligned}
$$

for all $t \in[0, T], m \geq 1$.

From (19) and (20), one determines that $t_{m}=$ $+\infty$, and the existence of a

$$
u \in L^{\infty}(0, T ; H) \cap L^{2}(0, T ; V) \quad \forall T>0,
$$

and a subsequence of $\left\{u_{m}\right\}_{m \in \mathbb{N}}$ which converges weak-star to $u$ in $L^{\infty}(0, T ; H)$ and weakly to $u$ in $L^{2}(0, T ; V)$ for all $T>0$. By the compactness Theorem 5.1 in Chapter 1 of [Lions, 1969], one can then deduce that a subsequence in fact converges strongly to $u$ in $L^{2}(0, T ; H)$ and a.e. in $(0, T) \times \Omega$ for all $T>0$. But the weak convergence in $L^{2}(0, T ; V)$ is not enough to ensure that

$$
\left\|u_{m}\right\| \rightarrow\|u\|
$$

or at least

$$
F_{N}\left(\left\|u_{m}(t)\right\|\right) \rightarrow F_{N}(\|u(t)\|) \quad \text { for a.a. } t .
$$

Thus, we need to find a stronger estimate. We now take the inner product of the Galerkin ODE (17) with $A u_{m}$ and obtain

$$
\begin{gathered}
\frac{1}{2} \frac{\mathrm{d}}{\mathrm{d} t}\left\|u_{m}\right\|^{2}+\nu\left|A u_{m}\right|^{2}+b_{N}\left(u_{m}, u_{m}, A u_{m}\right) \\
=\left(G\left(t, u_{m}(t-\rho(t))\right), A u_{m}\right) .
\end{gathered}
$$

Obviously,

$$
\begin{aligned}
& \left|\left(G\left(t, u_{m}(t-\rho(t))\right), A u_{m}\right)\right| \leq \frac{\nu}{4}\left|A u_{m}\right|^{2} \\
& +\frac{\left|G\left(t, u_{m}(t-\rho(t))\right)\right|^{2}}{\nu}
\end{aligned}
$$

By (5) and Young's inequality, it follows

$$
\begin{aligned}
\left|b_{N}\left(u_{m}, u_{m}, A u_{m}\right)\right| & \leq \frac{N}{\left\|u_{m}\right\|} C_{2}\left\|u_{m}\right\|^{3 / 2}\left|A u_{m}\right|^{3 / 2} \\
& =N C_{2}\left\|u_{m}\right\|^{1 / 2}\left|A u_{m}\right|^{3 / 2} \\
& \leq \frac{\nu}{4}\left|A u_{m}\right|^{2}+C_{N}\left\|u_{m}\right\|^{2},
\end{aligned}
$$

with $C_{N}=\frac{27\left(N C_{2}\right)^{4}}{4 \nu^{3}}$.
Thus (21) simplifies to

$$
\begin{aligned}
\frac{d}{d t}\left\|u_{m}\right\|^{2}+\nu\left|A u_{m}\right|^{2} \leq & \frac{2}{\nu}\left|G\left(t, u_{m}(t-\rho(t))\right)\right|^{2} \\
& +2 C_{N}\left\|u_{m}\right\|^{2}
\end{aligned}
$$

Let us assume now that $u^{0} \in V$. Then, from (22) and the fact that

$$
\left\|u_{m}(0)\right\|=\left\|P_{m} u^{0}\right\| \leq\left\|u^{0}\right\|
$$

by the choice of the basis $\left\{e_{j}\right\}$ of $H$, one easily obtains that the sequence $\left\{u_{m}\right\}$ is bounded in $L^{\infty}(0, T ; V)$ and in $L^{2}(0, T ; D(A))$ for all $T>0$.

Then, observe that for any $w \in H$, $\left|b_{N}\left(u_{m}, u_{m}, w\right)\right| \leq N C_{3}\left|A u_{m}\right||w|$, and in consequence, the sequence $\left\{P_{m} B_{N}\left(u_{m}, u_{m}\right)\right\}$ is bounded in $L^{2}(0, T ; H)$ for all $T>0$.

Therefore, from the equation

$$
\begin{aligned}
\frac{\mathrm{d} u_{m}}{\mathrm{~d} t}=- & \nu A u_{m}-P_{m} B_{N}\left(u_{m}, u_{m}\right) \\
& +P_{m} G\left(t, u_{m}(t-\rho(t))\right),
\end{aligned}
$$

it follows that the sequence $\left\{\frac{d u_{m}}{d t}\right\}$ is also bounded in $L^{2}(0, T ; H)$.

Consequently, as $D(A) \subset V \subset H$ with compact injection, by Theorem 5.1 in Chapter 1 of [Lions, 1969], there exists an element $u \in$ $L^{\infty}(0, T ; V) \cap L^{2}(0, T ; D(A))$ for all $T>0$, and a subsequence of $\left\{u_{m}\right\}$, that we will also denote by $\left\{u_{m}\right\}$, such that

$$
\left\{\begin{array}{l}
u_{m} \rightarrow u \quad \text { strong in } L^{2}(0, T ; V), \\
u_{m} \rightarrow u \quad \text { a.e. in }(0, T) \times \Omega \\
u_{m} \rightarrow u \quad \text { weak in } L^{2}(0, T ; D(A)), \\
u_{m} \stackrel{*}{*} \quad \text { weak-star in } L^{\infty}(0, T ; V), \\
\frac{\mathrm{d} u_{m}}{\mathrm{~d} t} \rightarrow \frac{\mathrm{d} u}{\mathrm{~d} t} \quad \text { weak in } L^{2}(0, T ; H),
\end{array}\right.
$$

for all $T>0$.

Also, as $u_{m}$ converges to $u$ in $L^{2}(0, T ; V)$ for all $T>0$, we can assume, possibly extracting a subsequence, that

$$
\left\|u_{m}(t)\right\| \rightarrow\|u(t)\| \quad \text { a.e. in }(0,+\infty),
$$

and therefore

$$
F_{N}\left(\left\|u_{m}(t)\right\|\right) \rightarrow F(\|u(t)\|) \quad \text { a.e. in }(0,+\infty) \text {. }
$$


From (23) and (24) we can take limits in (17) and we obtain that $u$ is a solution of (3) satisfying (16). In fact, this can be done reasoning as in [Caraballo et al., 2006] for the case without delays (see also [Lions, 1969] for the case of the NavierStokes system).

Assume now that $u^{0} \in H \backslash V$. Then, integrating in (22) between $s$ and $t$, for all $0 \leq s \leq t \leq T$, we obtain that

$$
\begin{aligned}
\left\|u_{m}(t)\right\|^{2} \leq & \left\|u_{m}(s)\right\|^{2} \\
& +\frac{2}{\nu} \int_{0}^{T}\left|G\left(r, u_{m}(r-\rho(r))\right)\right|^{2} d r \\
& +2 C_{N} \int_{0}^{T}\left\|u_{m}(r)\right\|^{2} d r \\
\leq & \left\|u_{m}(s)\right\|^{2} \\
& +\frac{2}{\nu\left(1-\rho_{*}\right)} \int_{-\rho(0)}^{0} \widetilde{g}(r)|\phi(r)|^{2} d r \\
& +\frac{2}{\nu\left(1-\rho_{*}\right)} \int_{0}^{T} \widetilde{g}(r)\left|u_{m}(r)\right|^{2} d r \\
& +\frac{2}{\nu} \int_{0}^{T} f(r) d r+2 C_{N} \int_{0}^{T}\left\|u_{m}(r)\right\|^{2} d r .
\end{aligned}
$$

Thanks to (19) and (20), we know that $u_{m}$ is bounded in $L^{2}(0, T ; V) \cap L^{\infty}(0, T ; H)$ and, consequently, there exists $\widetilde{K}_{T}>0$ such that

$$
\begin{aligned}
& \frac{2}{\nu\left(1-\rho_{*}\right)} \int_{-\rho(0)}^{0} \widetilde{g}(r)|\phi(r)|^{2} d r \\
& \quad+\frac{2}{\nu\left(1-\rho_{*}\right)} \int_{0}^{T} \widetilde{g}(r)\left|u_{m}(r)\right|^{2} d r \\
& \quad+\frac{2}{\nu} \int_{0}^{T} f(r) d r+2 C_{N} \int_{0}^{T}\left\|u_{m}(r)\right\|^{2} d r \\
& \leq \widetilde{K}_{T},
\end{aligned}
$$

for all integer $m \geq 1$.

Integrating now inequality (25) with respect to $s$ in the interval $[0, t]$, we have

$$
\begin{aligned}
t\left\|u_{m}(t)\right\|^{2} & \leq \int_{0}^{T}\left\|u_{m}(s)\right\|^{2} d s+T \widetilde{K}_{T} \\
& \leq \sup _{m \geq 1}\left(\int_{0}^{T}\left\|u_{m}(s)\right\|^{2} d s\right)+T \widetilde{K}_{T}:=\widehat{K}_{T},
\end{aligned}
$$

whence

$$
\left\|u_{m}(t)\right\|^{2} \leq \frac{1}{\varepsilon} \widehat{K}_{T}
$$

for all $t \in[\varepsilon, T]$, and all $0<\varepsilon<T$.
From (19), (26) and (22), we immediately obtain that the sequence $\left\{u_{m}\right\}$ is bounded in $L^{\infty}(0, T ; H)$, in $L^{2}(0, T ; V)$, in $L^{\infty}(\varepsilon, T ; V)$, and in $L^{2}(\varepsilon, T ; D(A))$, for all $T>\varepsilon>0$.

Reasoning as before (i.e. when $u^{0} \in V$ ), we see that the sequence $\left\{\frac{d u_{m}}{d t}\right\}$ is also bounded in $L^{2}(\varepsilon, T ; H)$ for all $T>\varepsilon>0$. Hence, there exists an element

$$
\begin{aligned}
& u \in L^{\infty}(0, T ; H) \cap L^{2}(0, T ; V) \cap \\
& \quad \cap L^{\infty}(\varepsilon, T ; V) \cap L^{2}(\varepsilon, T ; D(A))
\end{aligned}
$$

for all $T>\varepsilon>0$, and a subsequence of $\left\{u_{m}\right\}$, that we will also denote by $\left\{u_{m}\right\}$, such that

$$
\left\{\begin{array}{l}
u_{m} \rightarrow u \quad \text { weak in } L^{2}(0, T ; V), \\
u_{m} \stackrel{*}{\rightarrow} u \text { weak-star in } L^{\infty}(0, T ; H), \\
u_{m} \rightarrow u \quad \text { strong in } L^{2}(\varepsilon, T ; H), \\
u_{m} \rightarrow u \quad \text { a.e. in }(0, T) \times \Omega, \\
u_{m} \rightarrow u \quad \text { strong in } L^{2}(\varepsilon, T ; V), \\
u_{m} \rightarrow u \quad \text { weak in } L^{2}(\varepsilon, T ; D(A)), \\
u_{m} \stackrel{*}{\rightarrow} \quad \text { weak-star in } L^{\infty}(\varepsilon, T ; V), \\
\frac{d u_{m}}{d t} \rightarrow \frac{d u}{d t} \quad \text { weak in } L^{2}(\varepsilon, T ; H),
\end{array}\right.
$$

for all $T>\varepsilon>0$.

Also, as $u_{m}$ converges to $u$ in $L^{2}(\varepsilon, T ; V)$ for all $T>\varepsilon>0$, we can assume, eventually extracting a subsequence, that (24) is also satisfied in this case. From (27) and (24) we can take limits in (17) and we obtain that $u$ is a solution of (3) satisfying (15).

\section{Asymptotic behaviour of solutions}

In this section we obtain a result about the asymptotic behavior of the solutions of problem (3) when $t$ goes to $+\infty$.

Let us suppose that c1)-c3) hold with $g \in$ $L^{\infty}(\mathbb{R})$, assume also that

$$
\nu^{2} \lambda_{1}^{2}\left(1-\rho_{*}\right)>|g|_{\infty},
$$

where $|g|_{\infty}:=\|g\|_{L^{\infty}(\mathbb{R})}$,

and let us denote by $\varepsilon>0$ the unique solution of

$$
\varepsilon-\nu \lambda_{1}+\frac{|g|_{\infty} e^{\varepsilon h}}{\nu \lambda_{1}\left(1-\rho_{*}\right)}=0 .
$$


We can now formulate the following result.

Theorem 4.1. Under the previous assumptions, for any $\left(u^{0}, \phi\right) \in H \times L^{2}(-h, 0 ; H)$, and any $\tau \in \mathbb{R}$, the corresponding solution $u\left(t ; \tau, u^{0}, \phi\right)$ of problem (3) satisfies

$$
\begin{aligned}
& \left|u\left(t ; \tau, u^{0}, \phi\right)\right|^{2} \\
\leq & \left(\left|u^{0}\right|^{2}+\frac{|g|_{\infty} e^{\varepsilon h}}{\nu \lambda_{1}\left(1-\rho_{*}\right)} \int_{-h}^{0} e^{\varepsilon s}|\phi(s)|^{2} d s\right) e^{\varepsilon(\tau-t)} \\
& +\frac{e^{-\varepsilon t}}{\nu \lambda_{1}} \int_{\tau}^{t} e^{\varepsilon s} f(s) d s
\end{aligned}
$$

for all $t \geq \tau$.

In particular, if $\int_{\tau}^{\infty} e^{\varepsilon s} f(s) d s<\infty$, then every solution $u\left(t ; \tau, u^{0}, \phi\right)$ of (3) converges exponentially to 0 as $t \rightarrow+\infty$.

Proof. Let $u(t)=u\left(t ; \tau, u^{0}, \phi\right)$ be the solution of problem (3) corresponding to the initial data $\tau$, $u^{0}, \phi$. From

$$
\frac{1}{2} \frac{\mathrm{d}}{\mathrm{d} t}|u|^{2}+\nu\|u\|^{2}=(G(t, u(t-\rho(t))), u),
$$

we obtain,

$$
\begin{aligned}
& \frac{\mathrm{d}}{\mathrm{d} t}\left(e^{\varepsilon t}|u(t)|^{2}\right)=\varepsilon e^{\varepsilon t}|u(t)|^{2}-2 \nu e^{\varepsilon t}\|u(t)\|^{2} \\
& +2 e^{\varepsilon t}(G(t, u(t-\rho(t))), u) \\
& \leq\left(\varepsilon-\nu \lambda_{1}\right) e^{\varepsilon t}|u(t)|^{2} \\
& +\frac{1}{\nu \lambda_{1}} e^{\varepsilon t}|G(t, u(t-\rho(t)))|^{2}
\end{aligned}
$$

Now, observe that for any $t \geq \tau$,

$$
\begin{aligned}
& \int_{\tau}^{t} e^{\varepsilon s}|G(s, u(s-\rho(s)))|^{2} d s \\
\leq & |g|_{\infty} \int_{\tau}^{t} e^{\varepsilon s}|u(s-\rho(s))|^{2} d s+\int_{\tau}^{t} e^{\varepsilon s} f(s) d s,
\end{aligned}
$$

and

$$
\begin{aligned}
& \int_{\tau}^{t} e^{\varepsilon s}|u(s-\rho(s))|^{2} d s \\
& \leq \frac{e^{\varepsilon h}}{1-\rho_{*}} \int_{\tau}^{t} e^{\varepsilon s}|u(s)|^{2} d s \\
& +\frac{e^{\varepsilon h}}{1-\rho_{*}} \int_{\tau-h}^{\tau} e^{\varepsilon s}|\phi(s-\tau)|^{2} d s \\
& =\frac{e^{\varepsilon h}}{1-\rho_{*}} \int_{\tau}^{t} e^{\varepsilon s}|u(s)|^{2} d s \\
& +\frac{e^{\varepsilon(h+\tau)}}{1-\rho_{*}} \int_{-h}^{0} e^{\varepsilon s}|\phi(s)|^{2} d s .
\end{aligned}
$$

Integrating in (30), from (28), (31) and (32), we easily obtain (29).

\section{Pullback attractors}

\subsection{Preliminaries on pullback attractors}

We now recall some results on the theory of pullback attractors as developed in [Crauel et al., 1995], [Kloeden \& Stonier, 1998] and [Kloeden \& Schmalfuß]. It is a well known fact in dealing with non-autonomous problems, that the initial time is as important as the final one, yielding to the necessity of considering a two-parameter semigroup, a cocycle or a skew-product semiflow to set the problem in suitable framework. We will use the framework of two-parameter semigroups or evolution processes.

Definition 5.1. Let $X$ be a metric space. A family of mappings $\{U(t, \tau): X \rightarrow X: t, \tau \in \mathbb{R}, t \geq \tau\} \quad$ is said to be a process (or a two parameter semigroup, or an evolution semigroup) in $X$ if

$$
\begin{aligned}
U(t, r) U(r, \tau) & =U(t, \tau) & & \text { for all } t \geq r \geq \tau, \\
U(\tau, \tau) & =I d & & \text { for all } \tau \in \mathbb{R} .
\end{aligned}
$$

The process $U(\cdot, \cdot)$ is said to be continuous if the mapping $x \rightarrow U(t, \tau) x$ is continuous on $X$ for all $t, \tau \in \mathbb{R}, t \geq \tau$.

Recall that $\operatorname{dist}(A, B)$ denotes the Hausdorff semidistance between the sets $A$ and $B$, which is given by

$$
\operatorname{dist}(A, B)=\sup _{a \in A} \inf _{b \in B} d(a, b), \quad \text { for } A, B \subset X .
$$

Definition 5.2. Let $U(\cdot, \cdot)$ be a process in the metric space $X$. A family of compact sets $\{\mathcal{A}(t)\}_{t \in \mathbb{R}}$ is said to be a (global) pullback attractor for $U(\cdot, \cdot)$ if, for every $t \in \mathbb{R}$, if follows

(i) $U(t, \tau) \mathcal{A}(\tau)=\mathcal{A}(t) \quad$ for all $\tau \leq t$ (invariance), and

(ii) $\lim _{\tau \rightarrow-\infty} \operatorname{dist}(U(t, \tau) D, \mathcal{A}(t))=0$ (pullback attraction) for all bounded subset $D \subset X$. 
The concept of pullback attractor is related to that of pullback absorbing set.

Definition 5.3. The family of subsets $\{B(t)\}_{t \in \mathbb{R}}$ of $X$ is said to be pullback absorbing with respect to the process $U(\cdot, \cdot)$ if, for every $t \in \mathbb{R}$ and all bounded subset $D \subset X$, there exists $\tau_{D}(t) \leq t$ such that

$$
U(t, \tau) D \subset B(t), \text { for all } \tau \leq \tau_{D}(t) .
$$

In fact, as happens in the autonomous case, the existence of compact pullback attracting sets is enough to ensure the existence of pullback attractors. The following result can be found in [Crauel et al., 1995] and [Schmalfuß, 1992] (see also [Caraballo \& Real, 2004]).

Theorem 5.4. Let $U(\cdot, \cdot)$ be a continuous process on the metric space $X$. If there exists a family of compact pullback attracting sets $\{B(t)\}_{t \in \mathbb{R}}$, then there exists a pullback attractor $\{\mathcal{A}(t)\}_{t \in \mathbb{R}}$, with $\mathcal{A}(t) \subset B(t)$ for all $t \in \mathbb{R}$, given by

$$
\mathcal{A}(t)=\overline{\bigcup_{\substack{D \subset X \\ \text { bounded }}} \Lambda_{D}(t),}
$$

where

$$
\Lambda_{D}(t)=\bigcap_{n \in \mathbb{N}} \overline{\bigcup_{\tau \leq t-n} U(t, \tau) D} .
$$

\subsection{Existence of the pullback attractor for the GMNSED model}

We can now apply the theory of pullback attractors to analyse the asymptotic behaviour of our model (3) under appropriate assumptions.

\section{2(A). Construction of the associated process}

Now we will apply the theory in the previous section to prove the existence of an attractor for our nonautonomous GMNSE model with delay. To this end, we will consider that $G: \mathbb{R} \times H \rightarrow H$ satisfies c1), c2) and c3) with $g \in L^{\infty}(\mathbb{R})$. Thus, without loss of generality we can assume that $G$ satisfies c2) with $g \equiv 1$, and there exists a nonnegative constant $a$ such that

$$
|G(t, u)|^{2} \leq a|u|^{2}+f(t) \quad \forall(t, u) \in \mathbb{R} \times H .
$$

Under these assumptions, for each initial time $\tau \in \mathbb{R}$, and any $\phi \in C(-h, 0 ; H)$, Theorem 3.1 ensures that if we take $u^{0}=\phi(0)$, problem (3) possesses a unique solution

$$
u(\cdot ; \tau, \phi)=u(\cdot ; \tau, \phi(0), \phi),
$$

which belongs to the space $C([\tau-h, T] ; H) \cap$ $L^{2}(\tau, T ; V) \cap C([\tau+\epsilon, T] ; V) \cap L^{2}(\tau+\epsilon, T ; D(A))$ for all $T>\tau+\epsilon>\tau$.

Now, we proceed to construct the evolution process which can help us in the analysis of the long-time behaviour of our model. We define a process in the phase space $C_{H}=C([-h, 0] ; H)$ with sup norm, $\|\phi\|_{C_{H}}=\sup _{s \in[-h, 0]}|\phi(s)|$, as the family of mappings $U(t, \tau): C_{H} \rightarrow C_{H}$ given by

$$
U(t, \tau) \phi=u_{t}(\cdot ; \tau, \phi),
$$

for any $\phi \in C_{H}$, and any $\tau \leq t$, where $u_{t}(\cdot ; \tau, \phi) \in$ $C_{H}$ is defined by

$$
u_{t}(s ; \tau, \phi)=u(t+s ; \tau, \phi) \quad \forall s \in[-h, 0] .
$$

Now we will prove that $U(\cdot, \cdot)$ is a continuous process.

Proposition 5.5. Assume that $G$ satisfies c1), c2) with $g=1$, and that (33) also holds. Then, the family of mappings $U(\tau, t), \tau \leq t$, defined by (34) and (35) is a continuous process on $C_{H}$, and more exactly, for any pair $\phi, \psi \in C_{H}$ such that $\|\phi-\psi\|_{C_{H}} \leq$ 1, it follows

$$
\begin{aligned}
& \|U(t, \tau) \phi-U(t, \tau) \psi\|_{C_{H}}^{2} \\
& \leq\left(1+\frac{h}{1-\rho_{*}} L^{2}\left(R\left(t, \tau,\|\phi\|_{C_{H}}\right)\right)\right)\|\phi-\psi\|_{C_{H}}^{2} \\
& \times \exp \left\{\left(2 C_{3} N^{4}+1+\frac{L^{2}\left(R\left(t, \tau,\|\phi\|_{C_{H}}\right)\right)}{1-\rho_{*}}\right)(t-\tau)\right\},
\end{aligned}
$$

for all $t \geq \tau$, where $C_{3}>0$ is a constant only dependent on $\nu$ and the constant $C_{2}$ appearing in (6), and $R\left(t, \tau,\|\phi\|_{C_{H}}\right)$ is given by

$$
\begin{gathered}
R\left(t, \tau,\|\phi\|_{C_{H}}\right) \\
=\left\{\left(2+\frac{a h}{\nu \lambda_{1}\left(1-\rho_{*}\right)}\right)\left(1+\|\phi\|_{C_{H}}^{2}\right)\right. \\
\left.+\frac{1}{2 \nu \lambda_{1}} \int_{\tau}^{t} f(s) d s\right\}^{1 / 2} \times \\
\quad \times \exp \left\{\frac{a(t-\tau)}{4 \nu \lambda_{1}\left(1-\rho_{*}\right)}\right\}
\end{gathered}
$$


Proof. The uniqueness of solutions obviously implies that $U(\cdot, \cdot)$ is process.

We consider $\phi \in C_{H}$ and $\tau \in \mathbb{R}$ fixed. Let $\psi \in C_{H}$ such that $\|\phi-\psi\|_{C_{H}} \leq 1$. Denote by $u(\cdot)=u(\cdot ; \tau, \phi)$ and $v(\cdot)=u(\cdot ; \tau, \psi)$ the corresponding solutions to $(3)$.

Firstly, by (33) we have

$$
\begin{aligned}
& \frac{\mathrm{d}}{\mathrm{d} t}|v(t)|^{2}+2 \nu\|v(t)\|^{2} \\
& =2(G(t, v(t-\rho(t))), v(t)) \\
& \leq 2 \nu \lambda_{1}|v(t)|^{2}+\frac{1}{2 \nu \lambda_{1}}\left(a|v(t-\rho(t))|^{2}+f(t)\right),
\end{aligned}
$$

and therefore

$$
\begin{aligned}
|v(t)|^{2} & \\
\leq & |\psi(0)|^{2} \\
& +\frac{1}{2 \nu \lambda_{1}}\left(\frac{a h}{1-\rho_{*}}\|\psi\|_{C_{H}}^{2}+\int_{\tau}^{t} f(s) d s\right) \\
& +\frac{a}{2 \nu \lambda_{1}\left(1-\rho_{*}\right)} \int_{\tau}^{t}|v(s)|^{2} d s \\
\leq & \left(2+\frac{a h}{\nu \lambda_{1}\left(1-\rho_{*}\right)}\right)\left(1+\|\phi\|_{C_{H}}^{2}\right) \\
& +\frac{1}{2 \nu \lambda_{1}} \int_{\tau}^{t} f(s) d s \\
& +\frac{a}{2 \nu \lambda_{1}\left(1-\rho_{*}\right)} \int_{\tau}^{t}|v(s)|^{2} d s,
\end{aligned}
$$

for all $t \geq \tau$.

From this last inequality and Gronwall's lemma, we obtain

$$
|u(t ; \tau, \psi)|^{2} \leq R^{2}\left(t, \tau,\|\phi\|_{C_{H}}\right) \quad \forall t \geq \tau,
$$

and all $\phi$ and $\psi$ such that $\|\phi-\psi\|_{C_{H}} \leq 1$.

If we set $w=u-v$ and proceed as in the Proof of Theorem 2.3, we have that there exists a constant $C_{3}>0$ which depends on $C_{2}$ and $\nu$, such that,

$$
\begin{aligned}
& \frac{\mathrm{d}}{\mathrm{d} t}|w(t)|^{2} \leq\left(2 C_{3} N^{4}+1\right)|w(t)|^{2} \\
& \quad+|G(t, u(t-\rho(t)))-G(t, v(t-\rho(t)))|^{2} .
\end{aligned}
$$

Let us fix $T>\tau$. By (38) we know that $|u(t)| \leq$ $R\left(T, \tau,\|\phi\|_{C_{H}}\right)$, and $|v(t)| \leq R\left(T, \tau,\|\phi\|_{C_{H}}\right)$, for all $t \in[\tau, T]$, and by $(37)$ it is clear that $\|\phi\|_{C_{H}} \leq$ $R\left(T, \tau,\|\phi\|_{C_{H}}\right)$ and $\|\psi\|_{C_{H}} \leq R\left(T, \tau,\|\phi\|_{C_{H}}\right)$. Consequently, by $\mathrm{c} 2)$, one obtains that

$$
\begin{aligned}
& \int_{\tau}^{t}|G(s, u(s-\rho(s)))-G(s, v(s-\rho(s)))|^{2} d s \\
& \leq \frac{L^{2}\left(R\left(T, \tau,\|\phi\|_{C_{H}}\right)\right)}{1-\rho_{*}} \times \\
& \times\left(h\|\phi-\psi\|_{C_{H}}^{2}+\int_{\tau}^{t}|w(s)|^{2} d s\right),
\end{aligned}
$$

for all $t \in[\tau, T]$.

From (39), (40) and Gronwall's lemma, one can deduce

$$
\begin{aligned}
& |u(t ; \tau, \phi)-u(t ; \tau, \psi)|^{2} \\
& \leq\left(1+\frac{h}{1-\rho_{*}} L^{2}\left(R\left(t, \tau,\|\phi\|_{C_{H}}\right)\right)\right)\|\phi-\psi\|_{C_{H}}^{2} \\
& \times \exp \left\{\left(2 C_{3} N^{4}+1+\frac{L^{2}\left(R\left(t, \tau,\|\phi\|_{C_{H}}\right)\right)}{1-\rho_{*}}\right)(t-\tau)\right\},
\end{aligned}
$$

for all $t \geq \tau$.

Now, inequality (36) is an easy consequence of inequality (41), and the fact that for fixed $\tau$ and $\phi$, the right-hand member of this inequality is a nondecreasing function of $t$.

Finally, the continuity of $U(t, \tau)$ on $C_{H}$ is a direct consequence of (36).

\section{2(B). Existence of absorbing families of sets in $C_{H}$}

Now, we will prove that, under suitable assumptions, there exists a family of bounded pullback absorbing sets in $C_{H}$ for the process $U(t, \tau)$.

Assume that $G$ satisfies c1), c2) with $g=1$, (33), and

$$
\nu^{2} \lambda_{1}^{2}\left(1-\rho_{*}\right)>a .
$$

Let us denote $\varepsilon>0$ the unique solution of

$$
\varepsilon-\nu \lambda_{1}+\frac{a e^{\varepsilon h}}{\nu \lambda_{1}\left(1-\rho_{*}\right)}=0
$$

suppose that

$$
\int_{-\infty}^{0} e^{\varepsilon r} f(r) d r<\infty
$$

and define

$$
\rho_{H}(t)=1+\frac{e^{\varepsilon(1+h-t)}}{\nu \lambda_{1}} \int_{-\infty}^{t} e^{\varepsilon r} f(r) d r \quad t \in \mathbb{R} .
$$

We can now prove the following result. 
Theorem 5.6. Under the previous assumptions, the process $U(\tau, t), \tau \leq t$, defined by (34) and (35), satisfies

$$
\|U(t, \tau) \phi\|_{C_{H}}^{2} \leq \rho_{H}(t)
$$

for all $\tau \leq t-T_{D}$, and all $\phi \in D$, for any bounded $D \subset C_{H}$, where $T_{D}$ is defined by

$T_{D}=1+h$

$+\frac{1}{\varepsilon} \log \left[\left(1+\frac{a e^{\varepsilon h}}{\varepsilon \nu \lambda_{1}\left(1-\rho_{*}\right)}\right)\left(1+\sup _{\phi \in D}\|\phi\|_{C_{H}}^{2}\right)\right]$.

As a consequence, the family of closed balls $\left\{\bar{B}_{C_{H}}\left(0, \rho_{H}^{1 / 2}(t)\right)\right\}_{t \in \mathbb{R}}$ is pullback absorbing for the process $U(t, \tau)$.

Proof. With the same procedure as in the proof of Theorem 4.1, we obtain

$$
\begin{aligned}
& |u(t ; \tau, \phi)|^{2} \\
& \leq\left(|\phi(0)|^{2}+\frac{a e^{\varepsilon h}}{\nu \lambda_{1}\left(1-\rho_{*}\right)} \int_{-h}^{0} e^{\varepsilon r}|\phi(r)|^{2} d r\right) e^{\varepsilon(\tau-t)} \\
& +\frac{e^{-\varepsilon t}}{\nu \lambda_{1}} \int_{\tau}^{t} e^{\varepsilon r} f(r) d r
\end{aligned}
$$

and therefore

$$
\begin{aligned}
|u(t ; \tau, \phi)|^{2} & \\
\leq & \left(1+\frac{a e^{\varepsilon h}}{\varepsilon \nu \lambda_{1}\left(1-\rho_{*}\right)}\right)\|\phi\|_{C_{H}}^{2} e^{\varepsilon(\tau-t)} \\
& +\frac{e^{-\varepsilon t}}{\nu \lambda_{1}} \int_{-\infty}^{t} e^{\varepsilon r} f(r) d r
\end{aligned}
$$

for all $t \geq \tau$ and any $\phi \in C_{H}$.

From (48) one deduces that

$$
\begin{aligned}
|u(s ; \tau, \phi)|^{2} & \\
\leq & \left(1+\frac{a e^{\varepsilon h}}{\varepsilon \nu \lambda_{1}\left(1-\rho_{*}\right)}\right)\|\phi\|_{C_{H}}^{2} e^{\varepsilon(1+h+\tau-t)} \\
& +\frac{e^{\varepsilon(1+h-t)}}{\nu \lambda_{1}} \int_{-\infty}^{t} e^{\varepsilon r} f(r) d r
\end{aligned}
$$

for all $t-1-h \geq \tau, s \in[t-h-1, t]$, and any $\phi \in C_{H}$.

From (49) we immediately obtain (46).

As a direct consequence of the preceding result, we get the existence of the family of bounded absorbing sets in $C_{H}$.

\section{2(C). Existence of an absorbing family of bounded sets in $C_{V}$}

We now prove the existence of an absorbing family of bounded sets in $C_{V}=C([-h, 0] ; V)$ and a necessary bound on the term $\int_{t+\theta_{1}}^{t+\theta_{2}}|A u(r)|^{2} d r$. We proceed in a similar way as we have already done in the previous subsection.

Theorem 5.7. Under the assumptions in Theorem 5.6, there exist two positive functions $\rho_{V}, F \in$ $C(\mathbb{R})$ such that for any bounded set $D \subset C_{H}$ and for any $t \in \mathbb{R}$,

$$
\|u(t ; \tau, \phi)\|^{2} \leq \rho_{V}(t) \forall \tau \leq t-T_{D}, \forall \phi \in D,
$$

and

$$
\begin{gathered}
\int_{t+\theta_{1}}^{t+\theta_{2}}|A u(r ; \tau, \phi)|^{2} d r \leq F(t) \\
\forall \tau \leq t-T_{D}-h, \forall \theta_{1} \leq \theta_{2} \in[-h, 0], \quad \forall \phi \in D,
\end{gathered}
$$

where $T_{D}$ is given by (47).

Proof. Let us fix a bounded set $D \subset C_{H}, \phi \in D$, $t \in \mathbb{R}$, and $\tau \leq t-T_{D}$ (observe that in particular $\tau \leq t-1-h)$.

Let us denote

$$
u(r)=u(r ; \tau, \phi), \quad \forall r \in[t-1-h, t] .
$$

Evidently, we have

$$
\begin{aligned}
& \frac{\mathrm{d}}{\mathrm{d} t}|u(r)|^{2}+2 \nu\|u(r)\|^{2} \\
&= 2(G(r, u(r-\rho(r))), u(r)) \\
& \leq \nu \lambda_{1}|u(r)|^{2} \\
& \quad+\frac{1}{\nu \lambda_{1}}|G(r, u(r-\rho(r)))|^{2} \\
& \leq \nu\|u(r)\|^{2}+\frac{1}{\nu \lambda_{1}}\left(a|u(r-\rho(r))|^{2}+f(r)\right),
\end{aligned}
$$

and therefore, integrating between $t-1$ and $t$, we get

$$
\begin{aligned}
& |u(t)|^{2}+\nu \int_{t-1}^{t}\|u(r)\|^{2} d r \\
\leq & |u(t-1)|^{2}+\frac{a}{\nu \lambda_{1}\left(1-\rho_{*}\right)} \int_{t-1-h}^{t}|u(r)|^{2} d r \\
& +\frac{1}{\nu \lambda_{1}} \int_{t-1}^{t} f(r) d r .
\end{aligned}
$$


From this inequality, taking into account (46), we obtain that

$$
\begin{gathered}
\int_{t-1}^{t}\|u(r ; \tau, \phi)\|^{2} d r \leq I_{V}(t), \\
\forall \tau \leq t-T_{D}, \quad \forall \phi \in D,
\end{gathered}
$$

where

$$
\begin{aligned}
I_{V}(t)= & \left(1+\frac{a(h+1)}{\nu \lambda_{1}\left(1-\rho_{*}\right)}\right) \rho_{H}(t) \\
& +\frac{1}{\nu \lambda_{1}} \int_{t-1}^{t} f(r) d r .
\end{aligned}
$$

On the other hand, from the equality

$$
\begin{aligned}
& \frac{\mathrm{d}}{\mathrm{d} t}\|u(r)\|^{2}+2 \nu|A u(r)|^{2}+2 b_{N}(u(r), u(r), A u(r)) \\
& \quad=2(G(r, u(r-\rho(r))), A u(r))
\end{aligned}
$$

and the inequalities

$$
\begin{aligned}
& \left|2 b_{N}(u(r), u(r), A u(r))\right| \\
& \quad \leq 2 N C_{2}\|u(r)\|^{1 / 2}|A u(r)|^{3 / 2} \\
& \quad \leq \frac{\nu}{2}|A u(r)|^{2}+C^{(N)}\|u(r)\|^{2},
\end{aligned}
$$

with $C^{(N)}=27 N^{4} C_{2}^{4}\left(2 \nu^{3}\right)^{-1}$, and

$$
\begin{aligned}
& |2(G(r, u(r-\rho(r))), A u(r))| \\
& \quad \leq \frac{\nu}{2}|A u(r)|^{2} \\
& \quad+\frac{2}{\nu}\left(a|u(r-\rho(r))|^{2}+f(r)\right),
\end{aligned}
$$

we get

$$
\begin{aligned}
& \frac{\mathrm{d}}{\mathrm{d} t}\|u(r)\|^{2}+\nu|A u(r)|^{2} \\
& \leq C^{(N)}\|u(r)\|^{2}+\frac{2}{\nu}\left(a|u(r-\rho(r))|^{2}+f(r)\right) .
\end{aligned}
$$

From this last inequality we obtain

$$
\begin{aligned}
\|u(t)\|^{2} \leq & \|u(s)\|^{2}+C^{(N)} \int_{t-1}^{t}\|u(r)\|^{2} d r \\
& +\frac{2 a}{\nu\left(1-\rho_{*}\right)} \int_{t-1-h}^{t}|u(r)|^{2} d r \\
& +\frac{2}{\nu} \int_{t-1}^{t} f(r) d r \quad \forall s \in[t-1, t],
\end{aligned}
$$

and therefore, by (46) and (52), we deduce

$$
\begin{aligned}
\|u(t)\|^{2} \leq & \|u(s)\|^{2}+C^{(N)} I_{V}(t) \\
& +\frac{2 a(h+1)}{\nu\left(1-\rho_{*}\right)} \rho_{H}(t) \\
& +\frac{2}{\nu} \int_{t-1}^{t} f(r) d r \quad \forall s \in[t-1, t] .
\end{aligned}
$$

Integrating in $s$, and using again (52), we obtain (50), with

$$
\begin{aligned}
\rho_{V}(t) & =\left(1+C^{(N)}\right) I_{V}(t)+\frac{2 a(h+1)}{\nu\left(1-\rho_{*}\right)} \rho_{H}(t) \\
& +\frac{2}{\nu} \int_{t-1}^{t} f(r) d r .
\end{aligned}
$$

For the proof of (51), observe that by (50), if $\tau \leq t-T_{D}-h$ and $s \in[t-h, t]$, then

$$
\|u(s ; \tau, \phi)\|^{2} \leq \rho_{V}(s) \quad \forall \phi \in D,
$$

and therefore

$$
\|u(s ; \tau, \phi)\|^{2} \leq \max _{r \in[t-h, t]} \rho_{V}(r)
$$

for all $\tau \leq t-T_{D}-h, \quad \forall s \in[t-h, t], \quad \forall \phi \in D$. Integrating in (54), we obtain

$$
\begin{aligned}
& \nu \int_{t+\theta_{1}}^{t+\theta_{2}}|A u(r)|^{2} d r \\
& \quad \leq\left\|u\left(t+\theta_{1}\right)\right\|^{2}+\int_{t+\theta_{1}}^{t+\theta_{2}}\left(C^{(N)}\|u(r)\|^{2}\right. \\
& \left.\quad+\frac{2}{\nu}\left(a|u(r-\rho(r))|^{2}+f(r)\right)\right) d r,
\end{aligned}
$$

for all $\theta_{1} \leq \theta_{2} \in[-h, 0]$, and therefore, by (46) and (56) we obtain (51), with

$$
\begin{aligned}
& F(t)=\nu^{-1}\left(1+h C^{(N)}\right) \max _{r \in[t-h, t]} \rho_{V}(r) \\
& +2 \nu^{-2}\left(a h \max _{r \in[t-h, t]} \rho_{H}(r)+\int_{t-h}^{t} f(r) d r\right) .
\end{aligned}
$$

\section{2(D). Existence of the pullback attractor}

Now, under an additional assumption, we can prove the existence of the pullback attractor. 
Theorem 5.8. Under the assumptions in Theorem 5.6, suppose moreover that

$$
\sup _{s \leq 0} e^{-\varepsilon s} \int_{-\infty}^{s} e^{\varepsilon r} f(r) d r<\infty .
$$

Then there exists a pullback attractor $\left\{\mathcal{A}_{C_{H}}(t)\right\}_{t \in \mathbb{R}}$ for the process $U(\cdot, \cdot)$ in $C_{H}$ defined by (34) and (35). Moreover, $\mathcal{A}_{C_{H}}(t)$ is a bounded subset of $C_{V}$ for any $t \in \mathbb{R}$.

Proof. Observe that as in particular $f$ is nonnegative and locally integrable, condition (58) is equivalent to

$$
\sup _{s \leq t} e^{-\varepsilon s} \int_{-\infty}^{s} e^{\varepsilon r} f(r) d r<\infty \quad \forall t \in \mathbb{R},
$$

or, also equivalently,

$$
\sup _{s \leq t} \int_{s-1}^{s} f(r) d r<\infty \quad \forall t \in \mathbb{R} .
$$

Thus, if we define

$$
\widetilde{\rho}_{V}(t)=\sup _{s \leq t} \rho_{V}(s), \quad t \in \mathbb{R}
$$

we have

$$
\rho_{V}(t) \leq \widetilde{\rho}_{V}(t)<\infty, \quad \forall t \in \mathbb{R} .
$$

Let us consider the family $\left\{B_{0}(t)\right\}_{t \in \mathbb{R}}$, where

$$
B_{0}(t)=\bar{B}_{C_{V}}\left(0 ; \widetilde{\rho}_{V}^{1 / 2}(t)\right) \quad t \in \mathbb{R}
$$

This is a family of bounded sets in $C_{V}$, which is pullback absorbing for $U(\cdot, \cdot)$. More exactly, by (56), we have that for any $t \in \mathbb{R}$ and all bounded $D \subset C_{H}$,

$$
U(t, \tau) D \subset B_{0}(t) \quad \forall \tau \leq t-T_{D}-h .
$$

For each $t \in \mathbb{R}$ the set $B_{0}(t)$ is in particular a bounded subset of $C_{H}$, thus, if we consider the family $\{B(t)\}_{t \in \mathbb{R}}$ given by

$$
B(t)=U\left(t, t-T_{B_{0}(t)}-h\right) B_{0}(t) \quad t \in \mathbb{R},
$$

by (59), we have

$$
B(t) \subset B_{0}(t) \quad \forall t \in \mathbb{R} .
$$

The family $\{B(t)\}_{t \in \mathbb{R}}$ is also pullback absorbing for the process $U(\cdot, \cdot)$. In fact, if $D \subset C_{H}$ is bounded, and $\tau \leq t-T_{B_{0}(t)}-h-T_{D}-h$, we get

$$
\begin{aligned}
& U(t, \tau) D \\
& =U\left(t, t-T_{B_{0}(t)}-h\right) U\left(t-T_{B_{0}(t)}-h, \tau\right) D \\
& \subset U\left(t, t-T_{B_{0}(t)}-h\right) B_{0}\left(t-T_{B_{0}(t)}-h\right) \\
& \subset U\left(t, t-T_{B_{0}(t)}-h\right) B_{0}(t)=B(t) .
\end{aligned}
$$

If we prove that each $B(t)$ is relatively compact in $C_{H}$, then $\{\overline{B(t)}\}_{t \in \mathbb{R}}$ (where the closure is taken in $C_{H}$ ) is a family of compact pullback absorbing sets in $C_{H}$ for $U(\cdot, \cdot)$, what ensures the existence of the pullback attractor $\left\{\mathcal{A}_{C_{H}}(t)\right\}_{t \in \mathbb{R}}$ for this process, with $\mathcal{A}_{C_{H}}(t) \subset \overline{B(t)} \subset B_{0}(t)$ for all $t \in \mathbb{R}$.

Let us now prove this compactness property. To this end, we will use the Ascoli-Arzelà theorem, in other words, we have to check that for each $t \in \mathbb{R}$,

(A) The set $U\left(t, t-T_{B_{0}(t)}-h\right) B_{0}(t)$ is equicontinuous (i.e. $\forall \varepsilon>0, \exists \delta>0$ such that if $-h \leq \theta_{1} \leq \theta_{2} \leq 0$, with $\theta_{2}-\theta_{1} \leq \delta$, then

$$
\begin{aligned}
& \mid\left(U\left(t, t-T_{B_{0}(t)}-h\right) \phi\right)\left(\theta_{2}\right) \\
& \quad-\left(U\left(t, t-T_{B_{0}(t)}-h\right) \phi\right)\left(\theta_{1}\right) \mid \leq \varepsilon
\end{aligned}
$$

for all $\left.\phi \in B_{0}(t)\right)$.

(B) For each $\theta \in[-h, 0]$,

$$
\bigcup_{\phi \in B_{0}(t)}\left(U\left(t, t-T_{B_{0}(t)}-h\right) \phi\right)(\theta)
$$

is a relatively compact set in $H$.

Property (B) follows from (61) and the compactness of the injection of $V$ into $H$.

Finally, in order to prove (A) we fix $\phi \in B_{0}(t)$, and $-h \leq \theta_{1} \leq \theta_{2} \leq 0$. Let us denote $u(r)=u(r ; t-$ $\left.T_{B_{0}(t)}-h, \phi\right), r \in[t-h, t]$. Then, taking into account 
(46) and (56), we have

$$
\begin{aligned}
&\left|u\left(t+\theta_{1}\right)-u\left(t+\theta_{2}\right)\right| \\
&=\left|\int_{t+\theta_{1}}^{t+\theta_{2}} u^{\prime}(r) \mathrm{d} r\right| \\
& \leq \int_{t+\theta_{1}}^{t+\theta_{2}}\left|u^{\prime}(r)\right| \mathrm{d} r \\
& \leq \int_{t+\theta_{1}}^{t+\theta_{2}}\left(\nu|A u(r)|+\left|B_{N}(u(r), u(r))\right|\right. \\
&+|G(r, u(r-\rho(r)))|) \mathrm{d} r \\
& \leq \int_{t+\theta_{1}}^{t+\theta_{2}}\left(\nu|A u(r)|+c_{1}|A u(r)|\|u(r)\|\right. \\
&\left.+a^{1 / 2}|u(r-\rho(r))|+f^{1 / 2}(r)\right) \mathrm{d} r \\
& \leq \int_{t+\theta_{1}}^{t+\theta_{2}}\left(\left(\nu+c_{1} \widetilde{\rho}_{V}^{1 / 2}(t)\right)|A u(r)|\right. \\
&\left.+a^{1 / 2} \tilde{\rho}_{H}^{1 / 2}(t)+f^{1 / 2}(r)\right) \mathrm{d} r,
\end{aligned}
$$

and, consequently, by the Cauchy inequality and (51),

$$
\begin{aligned}
& \left|u\left(t+\theta_{1}\right)-u\left(t+\theta_{2}\right)\right| \\
& \leq\left\{\left(\nu+c_{1} \tilde{\rho}_{V}^{1 / 2}(t)\right) F^{1 / 2}(t)\right. \\
& \left.\quad+\left(\int_{t-h}^{t} f(r) d r\right)^{1 / 2}\right\}\left(\theta_{2}-\theta_{1}\right)^{1 / 2} \\
& \quad+a^{1 / 2} \tilde{\rho}_{H}^{1 / 2}(t)\left(\theta_{2}-\theta_{1}\right),
\end{aligned}
$$

which implies the needed equicontinuity.

The proof is now complete.

Acknowledgements. We would like to thank the referees for their interesting and helpful suggestions which allowed us to improve the results in the preliminary version of this paper.

This work has been partly supported by Ministerio de Ciencia e Innovación (Spain) under grant MTM2008-00088, and Junta de Andalucía (Spain) under grant P07-FQM02468.

\section{References}

Caraballo, T., Kloeden, P.E., \& Real, J. [2006] "Unique strong solutions and V-attractors of a three dimensional system of Globally Modified Navier-Stokes equations," Advanced Nonlinear Studies 6, 411-436.
Caraballo, T., Kloeden, P.E., \& Real, J. [2008] "Invariant measures and statistical solutions of the globally modified Navier-Stokes equations," Discrete Contin. Dyn. Syst. Ser. B, 10, 761-781.

Caraballo, T. \& Real, J. [2004] "Attractors for 2D-Navier-Stokes models with delays," J. Diff. Eqns. 205, 270-296.

Constantin, P. [2003] "Near identity transformations for the Navier-Stokes equations", in Handbook of Mathematical Fluid Dynamics, Vol. II, 117-141, North-Holland, Amsterdam.

Constantin, P. \& Foias, C. [1988] Navier-Stokes equations, Chicago Lectures in Mathematics. University of Chicago Press, Chicago.

Crauel, H., Debussche, A. \& Flandoli, F. [1995] "Random attractors," J. Dyn. Diff. Eq. 9, 307341.

Flandoli, F. \& Maslowski, B. [1995] "Ergodicity of the 2-D Navier-Stokes Equation under random perturbations," Commun. Math. Phys. 171, 119-141.

Hale, J.K. \& Verduyn Lunel, S.M. [1993] Introduction to Functional Differential Equations, Springer Verlag, New York.

Kloeden, P.E., Caraballo, T., Langa, J.A., Real, J. \& Valero, J. [2009] "The three dimensional globally modified Navier-Stokes equations," in Mathematical Problems in Engineering Aerospace and Sciences, Vol. 3, Chapter 2, Editors: S. Sivasundaram, J. Vasundhara Devi, Zahia Drici and Farzana Mcrae, Cambridge Scientific Publishers.

Kloeden, P.E., Langa, J.A. \& Real, J. [2007] "Pullback $V$-attractors of a three dimensional system of nonautonomous globally modified NavierStokes equations: Existence and finite fractal dimension," Commun. Pure Appl. Anal. 6, 937955 .

Kloeden, P.E., Marín-Rubio, P. \& Real, J. [2009] "Equivalence of Invariant measures and Stationary Statistical solutions for the autonomous globally modified Navier-Stokes equations," Commun. Pure Appl. Anal. 8, 785-802. 
Kloeden, P.E. \& Stonier, D.J. [1998] "Cocycle attractors in nonautonomously perturbed differential equations," Dynamics Continuous Discrete and Impulsive Systems 4, 211-226.

Kloeden, P.E. \& Schmalfuß, B. [1997] "Nonautonomous systems, cocycle attractors and variable time-step discretization," Numer. Algorithms 14, 141-152.

Kloeden, P.E. \& Valero, J. [2007] "The weak connectedness of the attainability set of weak solutions of the $3 D$ Navier-Stokes equations," Proc. Roy. Soc. London A 463, 1491-1508.

Lions, J.L. [1969] Quelques Méthodes de Résolution des Problèmes aux Limites Non Linéaires, Dunod, Paris.

Robinson, J.C. [2001] Infinite-dimensional dynamical systems, Cambridge University Press, Cambridge.

Romito, M. [2009] "The uniqueness of weak solutions of the Globally Modified Navier-Stokes equations," Advanced Nonlinear Studies, 9, 425427.

Schmalfuss, B. [1992] Backward cocycle and attractors of stochastic differential equations, in $V$. Reitmann, T. Redrich and N. J. Kosch (eds.), International Seminar on Applied MathematicsNonlinear Dynamics: Attractor Approximation and Global Behaviour, 185-192.

Temam, R. [1977] Navier-Stokes Equations, NorthHolland, Amsterdam.

Temam, R. [1995] Navier-Stokes Equations and Nonlinear Functional Analysis, Second Edition, SIAM, Philadelphia.

Yoshida, Z. \& Giga, Y. [1984] "A nonlinear semigroup approach to the Navier-Stokes system," Comm. in Partial Differential Equations 9, 215230 . 